\title{
Efeitos do contínuo de níveis de interferência contextual na aprendizagem do "putt" do golfe
}

CDD. 20.ed. 152.3

796.352

\author{
Gonçalo DIAS
}

Rui MENDES $S^{* * * * * *}$

\section{Resumo}

A estruturação da prática motora é uma variável de aprendizagem muito estudada na área de Controle Motor e Aprendizagem, sendo, neste âmbito, o efeito de Interferência Contextual um dos pressupostos teóricos mais investigados (TANI, 2005). Tendo como suporte a hipótese de PoRTER e MAGILL $(2004,2005)$, o objetivo deste estudo foi verificar se o grupo com prática num contínuo de níveis Interferência Contextual alcançava melhores resultados na aprendizagem do "putt", do golfe, comparativamente com os grupos de prática por blocos, em séries e aleatória. Participaram voluntariamente 48 estudantes ( 24 de cada gênero) do ensino superior com $21,2 \pm 1,4$ anos de idade, todos destros e inexperientes. Na fase de aquisição foram realizados 126 ensaios de "putt" às distâncias de 2, 2,75 e 3,5 metros do buraco. Vinte e quatro horas depois ocorreu a fase de retenção e o teste de tranferência. $\mathrm{Na}$ fase de retenção foram praticados 30 ensaios de forma aleatória. 0 teste de tranferência consistiu em 20 ensaios, 10 ensaios a 2,5 metros e 10 ensaios a 2,5 metros com 10 graus de desvio face ao centro do buraco. A análise dos resultados demonstra que não existem diferenças estatisticamente significativas entre grupos. 0 efeito do incremento de Interferência Contextual não foi verificado neste estudo.

UNITERMos: Interferência contextual; Prática; Golfe; Aquisição; Tranferência.

\section{Introdução}

Os investigadores da área de Controle Motor e Aprendizagem têm demonstrado particular interesse sobre o efeito de Interferência Contextual (IC) (BRADY, 2008; GuADAGNOLI \& LEE, 2004; TANI, 2005). Este fenômeno está relacionado com a influência que a estruturação da prática motora exerce na aprendizagem de habilidades motoras (e.g., BARREIROS, Figueiredo \& GODINHO, 2007; Brady, 1998; Magill, 2007; Magill \& Hall, 1990).

Os pressupostos teóricos que suportam o efeito de IC apontam para resultados positivos na fase de aquisição quando a prática é estruturada de forma menos variável - prática em blocos -, comparativamente a outras formas mais complexas de organização da prática. Em oposição, nos testes de retenção e transferência de aprendizagem, a prática com maior índice de instabilidade - prática aleatória - parece conduzir melhores níveis de desempenho motor (BATTig, 1966; SheA \& Morgan, 1979).

As duas hipóteses robustas que caracterizam o efeito de IC retratam as vantagens das atividades cognitivas de processamento de informação na aprendizagem (cf. MeIra Junior, Tani \& Manoel, 2001). Na linha de pensamento dos mesmos autores, constatamos que a hipótese da elaboração considera que mais de uma representação da habilidade motora pode ser armazenada na memória ativa e o executante compara cada variação e distingue uma da outra (Shea \& Zimny, 1983, 1988). Por sua vez, a hipótese da reconstrução parte do pressuposto que com a prática aleatória o executante reconstrói um plano de ação a cada tentativa, numa variação específica (LeE \& Magill, 1983, 1985).

Estudos recentes sobre o efeito de IC na Aprendizagem Motora resumem os resultados obtidos nas várias experiências efetuadas em contexto laboratorial e em situações reais de ensino-aprendizagem (e.g., Brady, 2008; Magill, 2007). As conclusóes destes trabalhos têm subjacente que o efeito de IC não se evidencia de forma inequívoca nas tarefas predominantemente abertas ou externamente reguladas que solicitam uma importante interação
*Faculdade de Ciências do Desporto e Educação Física, Universidade de Coimbra - Portugal.

**Escola Superior de Educação, Instituto Politécnico de Coimbra - Portugal.

${ }^{* * *}$ Centro Interdisciplinar e Estudo da Performance Humana - Portugal. 
de custos perceptivos motores (e.g., Figueiredo \& BARREIROS, 2001).

Genericamente, as razóes apresentadas para que o efeito de IC não possa ser considerado como um fenômeno global de aprendizagem extensível a todas as habilidades motoras estão relacionadas com as características, especificidade e grau de complexidade das tarefas, a organização e quantidade de prática implementada, a estrutura e organização das sessões de prática, o nível de experiência dos aprendizes e os aspectos manipulados (BARREIros, FigUeIREDO \& GodinHo, 2007; Brady, 2008; FigueIREdo, 2004).

Especificamente sobre o estudo do "putt" do golfe, autores como Porter e Magill (2004, 2005) defendem que pode ser benéfico promover um incremento gradual de IC, de forma a alcançar efeitos positivos, principalmente na fase de transferência. Esta forma de organização implica que no início da sessão de prática sejam usados ensaios estruturados da forma da prática em blocos seguidos da realização de ensaios organizados em séries e, por fim, os últimos ensaios da prática motora na fase de aquisição são produzidos em contexto de prática aleatória. Os resultados obtidos nestas experiências não confirmam este pressuposto de forma inequívoca, tornando inconclusiva e pouco evidente a exploração do incremento de níveis de IC ao longo da prática motora.

Face ao exposto, o presente trabalho teve como objetivo analisar o efeito do incremento de níveis de IC na aprendizagem do "putt". Pretendeu-se verificar se o efeito da prática organizada num incremento ou contínuo de IC ao longo da fase de aquisição se manifestava de forma positiva e significativa na melhoria do nível de desempenho motor, na retenção e transferência de aprendizagem, face à estruturação da prática organizada exclusivamente em blocos, aleatória ou em séries.

\section{Métodos}

\section{Amostra}

Quarenta e oito estudantes do ensino superior (24 do sexo feminino e 24 do sexo masculino), com 21,2 $\pm 1,4$ anos de idade, todos destros, sem experiência prévia relacionada com a prática de golfe, sendo divididos equitativamente por quatro grupos experimentais em termos de repartição dos dois sexos. Todos os sujeitos participaram como voluntários, assinando um termo de consentimento livre e esclarecido. O estudo foi realizado em conformidade com o código de ética da Universidade de Coimbra e as recomendações da Declaração de Helsínque em Pesquisa com Seres Humanos. Os participantes não sofriam de qualquer tipo de incapacidade física ou mental.

\section{Tarefa}

A tarefa adotada foi o "putt", do golfe, o que implicou o batimento de uma bola com um taco de comprimento padrão (tamanho 35), numa superfície horizontal e imóvel que se encontrava colocada no solo sobre uma rampa.

\section{Dispositivo experimental}

O dispositivo experimental incluiu um carpete artificial plano, usado por profissionais de Minigolfe, retangular, de cor verde, sem emendas, que se assemelhava à textura da superfície do "green" natural, com 10 metros de comprimento, dois metros de largura e quatro milímetros de espessura. Um "hole" real de golfe (buraco) foi colocado a $350 \mathrm{~cm}$ do limite do carpete e a $100 \mathrm{~cm}$ de cada extremidade lateral.

Foram marcados com giz cinco círculos do tamanho de bolas de golfe que assinalavam os locais donde se batia a bola. Os círculos estavam colocados de frente para o buraco a $100 \mathrm{~cm}$ de cada extremidade lateral do carpete. $\mathrm{O}$ círculo que se situava a 2,5 metros de distância, com a 10 graus de desvio do centro do buraco, foi utilizado para realizar o teste de transferência.

Por baixo do carpete foi colocada uma rampa com $100 \mathrm{~cm}$ de comprimento que elevava a superfície do carpete a $10 \mathrm{~cm}$ de altura, fazendo com que a bola subisse até ao nível de entrada do buraco. Unida à rampa estava uma plataforma com $400 \mathrm{~cm}$ de comprimento.

O dispositivo experimental continha seis réguas: duas de $150 \mathrm{~cm}$, duas de $100 \mathrm{~cm}$ e duas de $50 \mathrm{~cm}$ de comprimento. As réguas estavam posicionadas lateralmente, de modo a verificar os valores positivos e negativos em centímetros, do posicionamento da bola em relação ao buraco. 


\section{Procedimentos}

Foram consideradas duas sessões de prática. $\mathrm{Na}$ primeira, a fase de aquisição com 126 ensaios. $\mathrm{Na}$ segunda sessão, correspondente à fase de retenção e ao teste transferência, efetuaram-se 50 ensaios ( 30 de retenção e 20 de transferência). Um dia, i.e., 24 horas mediaram a realização entre a primeira e a segunda sessão.

Constituíram-se quatro grupos experimentais, de 12 elementos cada, seis por gênero. Os grupos de prática em blocos (GPB), prática aleatória (GPA), prática em séries (GPS) e prática em contínuo de níveis de IC (GPC) realizaram 126 ensaios na aquisição, batendo a bola a partir de três distâncias ( $2 \mathrm{~m}, 2,75 \mathrm{~m}$ e $3,5 \mathrm{~m}$ ) (FIGURA 1, representação gráfica da tarefa).
Identificando o momento em que ocorreu mudança no contínuo de IC, referimos que os primeiros 42 ensaios do GPC foram organizados através da prática em blocos, seguindo-se 42 ensaios de prática em séries e, a terceira e última sequência de ensaios (ensaio $85^{\circ}$ ao ensaio $126^{\circ}$ ), foi realizada com recurso à prática aleatória.

A ordem de distância de batimento foi contrabalançada no seio do grupo para os vários indivíduos. Por exemplo, os primeiros 42 ensaios de prática, quatro dos sujeitos do GPB realizaram o "putt" a 2 m, quatro indivíduos a 2,75 $\mathrm{m}$ e os restantes elementos a $3,5 \mathrm{~m}$.

$\mathrm{Na}$ fase de retenção, igual para todos os grupos, foram realizadas 30 ensaios de forma aleatória em 2 $\mathrm{m}$ (D1), 2,75 m (D2) e 3,5 m (D3). As siglas D1, D2 e D3 referem-se às distâncias entre os círculos desenhados no carpete e o centro do buraco.

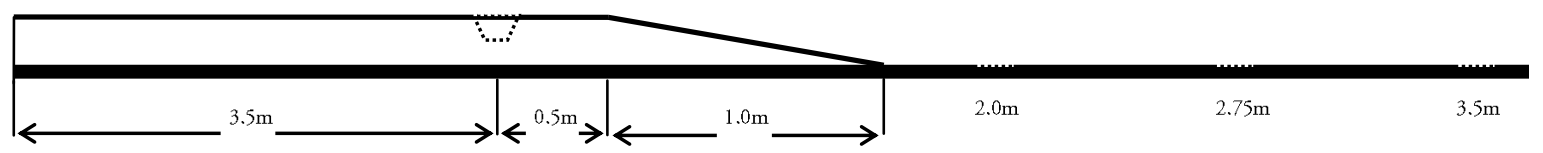

FIGURA 1 - Representação gráfica do dispositivo experimental: rampa, buraco e distâncias (vista lateral).

O teste de transferência consistiu na prática de 20 ensaios, sendo 10 ensaios realizados em D4 (2,5 m) e 10 ensaios em D5 (10 graus de desvio do buraco).

A tarefa realizou-se num espaço interior (pavilhão desportivo), onde os sujeitos foram analisados individualmente. Cada indivíduo foi informado sobre o objetivo do estudo, e no início da sessão prática a tarefa foi explicada e apresentada através de um filme-vídeo visionado num computador portátil, onde se enalteceram as quatro componentes técnicas fundamentais do "putt".

Após o visionamento do vídeo, o sujeito observou a demonstração efetuada pelo experimentador e realizou cinco ensaios de adaptação ao gesto, a 2,20 metros de distância do alvo, sendo corrigido pelo experimentador, no que às quatro componentes técnicas diz respeito. Depois, foram-lhe proporcionados mais três ensaios a 2,20 metros de distância do buraco para procurar acertar neste, em que não recebia qualquer correção ou informação por parte do experimentador.

Após a instrução e demonstração, era iniciada a fase de aquisição onde o indivíduo não recebeu informação verbal sobre a qualidade do movimento, nem sobre o resultado de cada ensaio (observava apenas o resultado). Imediatamente à realização de cada tentativa, era medido e registado o local onde a bola parava. De seguida, a bola era retirada da carpete pelo experimentador.

No dia a seguir à primeira sessão de prática foram realizados os testes de retenção e transferência.

A recolha de dados foi efetuada através da medição das distâncias de erro do comprimento e do erro lateral da bola face ao buraco. Os valores encontrados para o erro em comprimento $(\mathrm{C})$ e erro lateral (L), permitiram calcular o erro radial $(R)=\sqrt{ }(\mathrm{c} x$ $\mathrm{c}+\mathrm{l} \times \mathrm{l}$ ). Quando o sujeito acertava no buraco, o seu erro era zero (0), nos erros em comprimento e lateral e, por conseguinte, no erro radial.

A variável independente correspondeu à estruturação da prática motora utilizada: Prática em Blocos, Prática em Séries, Prática Aleatória e Prática organizada num contínuo de níveis de IC - GPB, GPS, GPA e GPC. As variáveis dependentes usadas foram o Erro Absoluto (EA) e Erro Variável (EV) calculadas em função do erro radial.

\section{Análise estatística}

Através da Análise da Variância com medidas repetidas no último fator, quatro grupos $(\mathrm{GPB}$, GPA, GPS e GPC) x nove blocos (A1, A2, A3, A4, A5, A6, A7, A8 e A9) foram analisados os resultados da aquisição. O teste de Tukey HSD foi aplicado 
para a análise de dados "Post Hoc", no sentido de determinar a existência de diferenças estatisticamente significativas entre grupos. Os dados da retenção e transferência foram analisados usando a Anova "One-Way". O nível de significância usado foi de 0,05. A análise estatística foi realizada no programa SPSS (versão 16).

\section{Resultados}

\section{Erro absoluto}

No que se refere à evolução do Erro Absoluto dos quatro grupos na fase de aquisição (FIGURA 2, blocos
Para efeitos de cálculo foram considerados nove blocos de ensaios na fase aquisição (A1, A2, A3, A4, A5, A6, A7, A8 e A9) cada um com 14 ensaios. $\mathrm{Na}$ fase de retenção foram calculados três blocos de ensaio, cada um com 10 ensaios (R1, R2 e R3) e no teste de transferência dois blocos de ensaios cada um com 10 ensaios (T1 e T2).

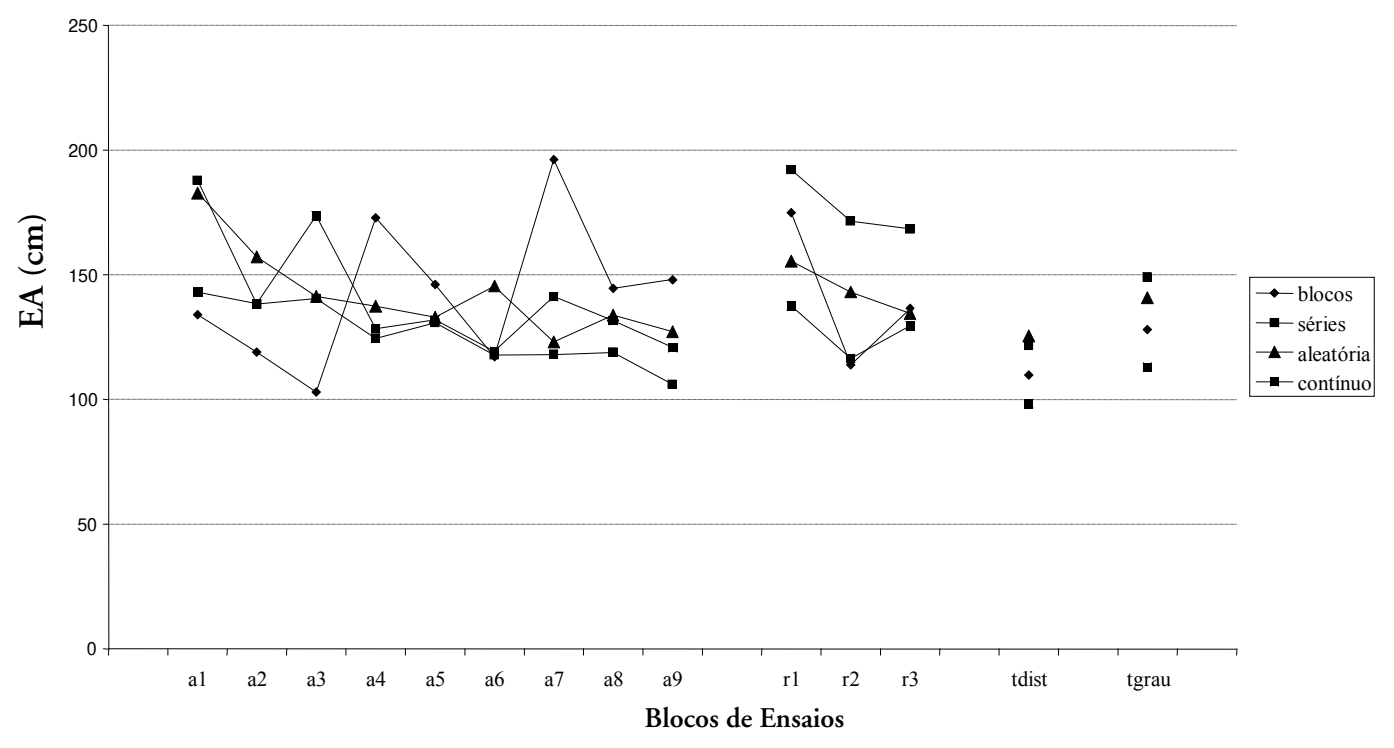

FIGURA 2 - Erro Absoluto (EA) médio por blocos de ensaios dos quatro grupos na aquisição, retenção e transferência.

A análise do desempenho na fase de aquisição revelou a existência de diferenças estatisticamente significativas entre diferentes blocos $(\mathrm{F}(8,352)=$ 9,053, $\mathrm{p} \leq 0,001)$. No GPB as diferenças entre blocos de ensaios são evidentes. No quarto (A4) e sétimo (A7) blocos de ensaios observou-se que a tendência de melhoria de desempenho nos blocos de ensaios anteriores (A2 e A3 para o bloco de ensaio A4, A5 e A6 para o bloco de ensaios A7) foi quebrada. Este fenômeno está relacionado com o fato dos sujeitos deste grupo mudarem de distâncias de batimento nestes momentos (A4 e A7), sendo, por conseguinte, previsível uma degradação do nível de desempenho motor. A análise da interação blocos com grupos apresentou diferenças com significado estatístico.
A1 a A9) foi observada uma tendência de pior desempenho do GPC face aos grupos GPS e GPB. Durante esta fase os quatro grupos experimentais não se diferenciaram significativamente $(F(3,44)=0,245, p=0,84)$. 


\section{Erro variável}

A evolução do Erro Variável (EV) dos quatro grupos na fase de aquisição (FIGURA 3, blocos A1 a A9) demonstrou que os grupos de prática aleatória, prática em blocos e a prática organizada num contínuo de níveis de IC apresentaram melhores resultados que o grupo de prática em séries. Não foram detectadas diferenças significativas entre grupos $(\mathrm{F}(3,44)=2,682, \mathrm{p}=0,058)$, entre blocos, $(\mathrm{F}(8,352)=1,498, \mathrm{p}=0,15)$, na interação entre grupos e blocos.

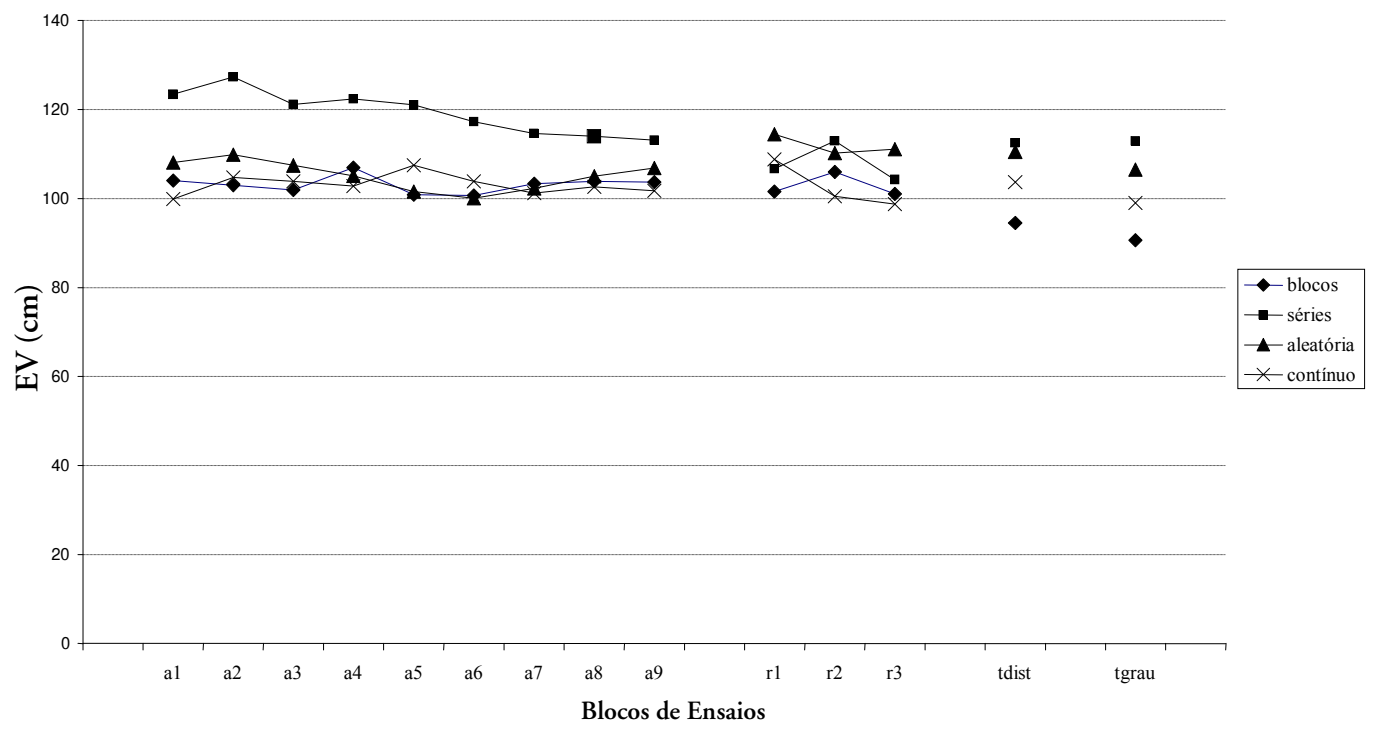

FIGURA 3 - Erro Variável (EV) por blocos de ensaios dos quatro grupos na aquisição, retenção e transferência.

Na retenção verificou-se que o GPC obteve melhor desempenho do que os restantes grupos, sendo que o grupo GPA revelou a pior prestação. Não se registaram diferenças significativas entre grupos nos três blocos da retenção: $\mathrm{R} 1-\mathrm{F}(3,44)=0,518, \mathrm{p}=$ $0,67, \mathrm{R}-2: \mathrm{F}(3,44)=0,740, \mathrm{p}=0,53, \mathrm{R} 3: \mathrm{F}(3,44)$ $=0,512, \mathrm{p}=0$.

\section{Discussão}

O pressuposto de incremento de IC ao longo do processo de prática motora, desenvolvido por PORTER e MAGILL $(2004,2005)$ foi, em termos teóricos, a base desta pesquisa. O presente estudo pretendia verificar se o efeito do incremento de IC se manifestava no "putt" do golfe. Tal como verificado num estudo recente de MEndes, Martins e Dias (2008) esperavase que grupo organizado num contínuo de níveis de IC obtivesse melhores resultados comparativamente com os grupos que praticaram sob a forma de blocos, séries e aleatória. Embora os resultados de EA obtidos na fase de transferência demonstrem uma tendência de superioridade do GPC em relação aos restantes grupos, estes não se traduziram em diferenças estatisticamente significativas.
Nos testes de transferência o grupo GPB obteve a melhor performance, seguindo-se o grupo GPC. Também nos dois testes de transferência não foram detectadas diferenças estatisticamente significativas entre diferentes grupos: transferência em distância, $\mathrm{F}(3,44)=1,384, \mathrm{p}=0$,26 e transferência em ângulo, $\mathrm{F}(3,44)=2,166, \mathrm{p}=0,10$.

O incremento gradual de IC aplicado através de um contínuo de variação da variabilidade da prática motora (GPC), por forma a conduzir a melhores performances ao nível do "putt", não resultou em melhores resultados na aprendizagem, em relação às clássicas formas de organização da prática motora no âmbito do estudo do efeito de interferência contextual. Assim, não se confirmou a hipótese anteriormente formulada quanto à ocorrência do efeito de IC em estudo.

A tarefa e o dispositivo experimental utilizados aproximam o "putt" da situação real da sua execução num jogo de golfe. De acordo com Brady (1998, 2008) vários fatores podem contribuir para que o efeito de IC não seja detectado em tarefas reais de 
ensino-aprendizagem (de campo), tais como: as características da amostra, a organização e quantidade de prática, a especificidade e o grau de complexidade da tarefa e a estrutura e organização das sessões.

A razão por não termos verificado diferenças estatisticamente significativas entre grupos, poder-se-á explicar em virtude de todos os sujeitos serem inexperientes. O fato da nossa amostra ser constituída por sujeitos principiantes ou inexperientes pode ter limitado ou condicionado a manifestação do efeito em estudo. Este fenômeno é referido por HEBERT, LANDin e Solmon (1996) que concluíram que o efeito de IC foi superior em indivíduos menos habilidosos quando comparados com sujeitos mais experientes, o que não se verificou no presente estudo.

A justificação anteriormente apresentada merece futura investigação, pois não é coincidente com as conclusões dos estudos de Guadagnoli, Holcomb e Weber (1999) e de Hall, Domingues e Cavazos (1994) respectivamente com o "putt" e o batimento de "basebol", onde o efeito de IC parece ser mais evidente quando os sujeitos da amostra são experientes.

A quantidade de prática é outra variável que pode ser relevante nos resultados obtidos em estudos laboratoriais e de campo. SHEa, KoHL e Indermill (1990); Prahl e EdWards (1995), advogam que um volume de prática mais elevado oferece maior probabilidade do efeito de IC ser detectado. Já para Hebert, Landin e Solmon (1996), tarefas de campo desta natureza parecem necessitar de uma quantidade assinalável de prática, para que os executantes atinjam elevados graus de proficiência, uma vez que estas podem tornar-se de difícil execução para sujeitos inexperientes.

Não obstante o que foi dito anteriormente, salientamos que os 12 estudos conhecidos que estudaram o efeito da variabilidade da prática na aprendizagem da tarefa "putt" do golfe também não permitem generalizar o efeito de IC no "putt", pois verificam-se resultados contraditórios na aprendizagem deste movimento (e.g., GoodwIN \& Meeuwsen, 1996; Guadagnoli, Holcomb \& Weber, 1999; Horner, Fitzpatrick \& SMyth, 2008; Hwang, Wright, McBride, Magnusson \& Buchanan 2004; Landin, Grisham \& BaUm, 2001; Mendes, Martins \& Dias, 2008; Porter, 2008; Porter, Landin, Hebert \& Baum, 2007; Porter \& Magill, 2007; Wu \& Magill, 2003).
No que se refere ao grau de complexidade da tarefa, podemos afirmar que esta é de difícil execução. Neste contexto, Pelz (2000) defende que o "putt" do golfe é uma habilidade motora que envolve uma série de variáveis que têm uma influência decisiva na sua aprendizagem, nomeadamente a concentração, a postura corporal, a rotina, a experiência e a amplitude do movimento.

Considerando as hipóteses explicativas que suportam o efeito de IC, pressupomos que a complexidade da habilidade motora "putt" poderá ter condicionado a representação mental do movimento na memória ativa dos executantes, influenciando o seu desempenho (SheA \& Zimny, 1983, 1988). Neste sentido, mesmo perante a prática com maior índice de variabilidade (e.g., aleatória), admitimos que os sujeitos podem não ter conseguido reconstruir um novo plano de ação a cada ensaio, limitando desta forma o seu sucesso na tarefa (LeE \& MAGILL, 1983, 1985).

Face aos resultados obtidos neste estudo, urge investigar os efeitos da prática estruturada num contínuo de níveis de IC, bem como o efeito de outro tipo de prática "mistas" na aprendizagem de habilidades motoras, por sujeitos inexperientes e compará-los com indivíduos com níveis de experiência mais elevados.

A eventual discordância entre os resultados conhecidos sobre os estudos de laboratório e os que usam tarefas reais de ensino-aprendizagem pode caminhar no sentido de realizar novas pesquisas orientadas para outras concepções teóricas, por forma a melhor compreender o papel da prática no processo de aprendizagem.

De salientar que a maioria dos trabalhos até agora publicados teve como pano de fundo um enfoque cognitivista. Assim, é recomendável que o efeito de IC seja analisado sob um outro prisma, de forma a integrar diferentes abordagens, nomeadamente, a Teoria de Sistemas Dinâmicos (cf. TANI, 2005).

Por último, tendo em conta o número limitado de estudos sobre o incremento de níveis de IC, importa clarificar e aprofundar este efeito ao nível da tarefa do "putt", recorrendo a outro tipo de amostras, tais como: crianças, idosos e pessoas com deficiência moderada, tanto mais que, recentemente, PORTER (2008) e Mendes, Martins e Dias (2008) verificaram efeitos positivos na realização da mesma tarefa estruturada num contínuo de níveis de IC. 


\section{Conclusão}

Os resultados obtidos permitem concluir que o efeito do contínuo de níveis de IC não foi confirmado no nosso estudo de forma evidente. Embora exista uma tendência de superioridade do grupo do GPC no teste de transferência, não foram verificadas diferenças estatisticamente significativas entre os vários grupos experimentais. Estes resultados confirmam as conclusões dos estudos de Porter e Magill $(2004,2005)$, Porter e Magill (2007), Horner, Fitzpatrick e Smyth (2008).

A combinação entre vários tipos de prática, num incremento ou contínuo de níveis de IC no decurso da prática, não resultou em efeitos positivos na aprendizagem do "putt". A complexidade da tarefa e os aspectos inerentes à realização da mesma podem ter produzido efeitos colaterais no nível de desempenho dos sujeitos, tal como defendem GuadaGNOLI e LEE (2004).

O efeito de níveis de IC não pode ser generalizável na aprendizagem de habilidades motoras, sendo oportuno aprofundar o referencial teórico já conhecido que o suporta e aumentar o número de pesquisas que permitam clarificar o impacto deste efeito na aprendizagem.

Perante estes fatos, importa estudar se a quantidade de prática, nesta tarefa e estruturada num contínuo de níveis de IC, se expressa em efeitos significativos, se não apenas na tendência observada de que o GPC apresenta melhores resultados que os restantes tipos de prática motora.

\section{Abstract}

Effects of a contextual interference continuum on golf putting task

The purpose of this study was to investigate the contextual interference $(\mathrm{Cl})$ effect on learning a golf putting task. PoRTER and MAGILL $(2004,2005)$ conducted studies where they applied a new concept of practice: the contextual interference continuum. The hypothesis is that practicing with gradual increases of $\mathrm{Cl}$ will lead to better performance on retention and transfer tests that both the blocked and random groups. Forty undergraduate students $(\mathrm{N}=48)$ were randomly assigned to one of four groups: blocked, serial, random, or increasing $\mathrm{Cl}$ practice schedule. Participants were inexperienced with the task and were blinded to the purpose of the experiment. During the acquisition they putted 126 trails from three distances (2, 2.75 and 3.5 meters) for the same golf hole target. In retention test they performed 30 trials in a random order. In the transfer test the subjects performed 20 trials: 10 at 2.5 meters and 10 to 2.5 meters with 10 degrees of deviation from the starting point towards the hole. The apparatus of this study consisted of one artificial putting indoor golf carpet. All participants putt the same golf ball with the same right-handed putter. The data of retention and transfer tests were analyzed using separate one-way ANOVAs. Although it is observed in the absolute error a trend of superiority of performance of the group or increasing $\mathrm{Cl}$ practice schedule on transfer test, the analysis didn't reveal a significant main effect for practice conditions. The effect of increasing $\mathrm{Cl}$ was not found in this experiment.

UnITERMS: Contextual interference; Practice; Golf; Acquisition; Transfer.

\section{Referências}

BARREIROS, J.; FIGUEIREDO, T.; GODINHO, M. The contextual interference effect in applied settings. European Physical Education Review, Manchester, v.81, p.195-208, 2007.

BATTIG, W.F. Facilitation and interference. In: BILODEAU, E.A. (Ed.). Acquisition of skill. New York: Academic Press, 1966. p.215-44.

BRADY, F. A theoretical and empirical review of the contextual interference effect and the learning of motor skills. Quest, Champaign, v.50. p.266-93, 1998. 
The contextual interference effect and sport skills. Perceptual and Motor Skills, Missoula, v.106, p.461-72, 2008. FIGUEIREDO, T. Interferência contextual em contexto aplicado. In: BARREIROS, J.; GODINHO, M.; MELO, F.; NETO, C. (Eds.). Desenvolvimento e aprendizagem: perspectivas cruzadas. Lisboa: Edições FMH, 2004. p.137-62. FIGUEIREDO, T.; BARREIROS, J. Interferência contextual numa tarefa de antecipação-coincidência com crianças. In: GUEDES, M.G.S. (Ed.). Aprendizagem motora: problemas e contextos. Lisboa: Edições FMH, 2001. p.79-92. GOODWIN, J.E.; MEEUWSEN, H.J. Investigation of the contextual interference effect in the manipulation of the motor parameter of the over-all force. Perceptual and Motor Skills, Missoula, v.83, p.735-43, 1996.

GUADAGNOLI, M.A.; LEE, T.D. Challenge point: a framework for conceptualizing the effects of various practice conditions in motor learning. Journal of Motor Behaviour, Washington, v.36, p.212-24, 2004.

GUADAGNOLI, M.A; HOLCOMB, W.R; WEBER, T.J. The relationship between contextual interference effects and performer expertise on the learning of putting task. Journal of Human Movement Studies, London, v.37, p.19-36, 1999. HALL, K.G.; DOMINGUES, D.A.; CAVAZOS, R. Contextual interference effects with skilled baseball players. Perceptual and Motor Skills, Missoula, v.78, p.835-41, 1994.

HEBERT, E.P.; LANDIN, D.; SOLMON, M.A. Practice schedule effects on the performance and learning of low-and high-skilled students: an applied study. Research Quarterly for Exercise and Sport, Washington, v.67, p.52-8, 1996.

HORNER, K.; FITZPATRICK, K.; SMYTH, P. The effect of increasing contextual interference on the practising of a motor skill. In: ANNUAL CONGRESS OF THE EUROPEAN COLLEGE OF SPORT SCIENCE, 13., Estoril, 2008. Book of Abstracts... Estoril, ECSS, 2008. p.A-71.

HWANG, G.Y.; WRIGHT, D.L.; MCBRIDE, R.; MAGNUSSON, C.E.; BUCHANAN, J. Experiencing greater contextual interference during practice impacts movement kinematics of the golf putt. Research Quarterly for Exercise and Sport, Washington, v.75, 2004. Supplement A-47.

LANDIN, D.; GRISHAM, W.; BAUM, B. Practice schedule effects on learning the golf putt and pitch. Research Quarterly for Exercise and Sport, Washington, p.49-50, 2001. Supplement 57.

LEE, T.D.; MAGILL, R.A. The locus of contextual interference in motor skill acquisition. Journal of Experimental Psychology: Learning, Memory and Cognition, Washington, v.9, p.730-46, 1983.

Can forgetting facilitate skill acquisition? In: GOODMAN, D.; WILBERG, R.B.; FRANKS, I.M. (Eds.). Differing perspectives in motor learning, memory, and control. Amsterdam: North-Holland, 1985. p.3-22.

MAGILL, R.A. Motor learning and control: concepts and applications. 8th ed. New York: McGraw-Hill, 2007.

MAGILL, R.A.; HALL, K.G. A review of the contextual interference effect in motor skill acquisition. Human Movement Science, Amsterdam, v.9, p.241-89, 1990.

MEIRA JUNIOR, C.M.; TANI, G.; MANOEL, E.J. A estrutura da prática variada em situações reais de ensino-aprendizagem. Revista Brasileira de Ciência e Movimento, São Caetano do Sul, v.9, p.55-63, 2001.

MENDES, R.; MARTINS, R.; DIAS, G. Effects of a contextual interference continuum on golf putting task. In: ANNUAL CONGRESS OF THE EUROPEAN COLLEGE OF SPORT SCIENCE, 13., Estoril, 2008. Book of Abstracts... Estoril: ECSS, 2008. p.A-490.

PELZ, D. Putting bible: the complete guide to mastering the green. New York: Doubleday, 2000.

PORTER, J.M. Systematically increasing contextual interference is beneficial for learning novel motor skills. Dissertation (Doctor of Philosophy) - Department of Kinesiology, Faculty of the Louisiana, 2008.

PORTER, J.M.; LANDIN, D.; HEBERT, E.P.; BAUM, B. The effects of three levels of contextual interference on performance outcomes and movement patterns in golf skills. International Journal of Sports Science \& Coaching, Leeds, v.2, p. 243-45, 2007. PORTER, J.M.; MAGILL, R.A. The effects of practicing a golf putting task moving along the contextual interference continuum. Journal of Sport \& Exercise Psychology, Champaign, v.26, p.S-151, 2004. Supplement.

Practicing along the contextual interference continuum increases performance of a golf putting task. Journal of Sport \& Exercise Psychology, Champaign, v.27, p.S-124, 2005. Supplement.

Practicing along the contextual interference continuum, a comparison of three practice schedules: An applied study. Medicine \& Science in Sports \& Exercise, Madison, 2007. Supplement. S-329.

PRAHL, B.K.; EDWARDS, W.H. A field test of the contextual interference effects on skill acquisition in pickle- ball with seventh grade boys and girls. Research Quarterly for Exercise and Sport, Washington, v.66, 1995. Supplement, A-55. SHEA, C.H.; KOHL, R.; INDERMILL, C. Contextual interference: contributions of practice. Acta Psychologica, Amsterdam, v.73, p.145-57, 1990.

SHEA, J.B.; MORGAN, R.L. Contextual interference effects on the acquisition, retention and transfer of motor skill. Journal of Experimental Psychology: Human Learning and Memory, Washington, v.5, p.178-87, 1979. 
SHEA, J.B.; ZIMNY, S.T. Context effects in memory and learning movement information. In: MAGILL, R.A. (Ed.). Memory and control of action. Amsterdam: North-Holland, 1983. p.345-66.

. Knowledge incorporation in motor representation. In: MEIJER, O.G.; ROTH, K. (Eds.). Complex movement behaviour: the motor-action controversy. Amsterdam: North-Holland, 1988. p.289-14.

TANI, G. Comportamento motor: aprendizagem e desenvolvimento. Rio de Janeiro: Guanabara Koogan, 2005.

WU, W.; MAGILL, R. A result-dependent practice schedule and learning a golf-putting skill. Journal of Sport \& Exercise Psychology, Champaign, v.25, p.S-141, 2003. Suplement (Presented at the 2003 North American Society for Psychology of Sport and Physical Activity Conference, NASPSPA, Sawannah).

\begin{tabular}{r|r} 
ENDEREço & \\
Gonçalo Dias & \\
Faculdade de Ciências do Desporto e Educação Física & Recebido para publicação: 15/03/2010 \\
Universidade de Coimbra & Revisado em: 09/06/2010 \\
Estádio Universitário - Pavilhão III & Aceito: 23/06/2010 \\
3040-156 - Coimbra - PORTUGAL & \\
e-mail: goncalodias@fcdef.uc.pt & \\
&
\end{tabular}

